Bio - grafía. Escritos sobre la Biología y su Enseñanza. ISSN 2027-1034

Edición Extraordinaria. p.p. 789 - 798

Memorias del IX Encuentro Nacional de Experiencias en Enseñanza de la Biología y la

Educación Ambiental. IV Congreso Nacional de Investigación en Enseñanza de la Biología.

\title{
LA CONT AMINACIÓN ATMOSFÉRICA: EL ONEPSI COMO ESTRATEGIA PARA ANALIZAR CAMBIOS EN LOS MODELOS DE LOS ESTUDIANTES A PARTIR DE UNA SECUENCIA DE ENSEÑANZA Y APRENDIZAJE \\ ATMOSPHERIC POLLUTION: A VIEW ON CHANGES IN STUDENTS 'MODELS FROM A SEQUENCE OF TEACHING AND LEARNING
}

\author{
Andrés González Hincapié \\ Fanny Angulo Delgado
}

RESUMEN:

La presente propuesta pertenece a una investigación ya finalizada, esta se acoge a la perspectiva centrada en la construcción de modelos y la modelización, en la cual se considera la enseñanza y el aprendizaje como una construcción dinámica tanto por parte del profesor como de los estudiantes. El propósito central es observar la evolución de los modelos construidos por estudiantiles por medio de la utilización de una secuencia de enseñanza y aprendizaje de un grupo estudiantes de grado octavo del colegio Tercer Milenio del municipio de Caldas (Antioquia). Se empleó una metodología de corte cualitativo, específicamente el estudio de caso instrumental. Los estudiantes construyeron modelos estudiantiles en relación al conocimiento científico, que reflexionen sobre el equilibrio ecológico - para el caso de la contaminación atmosférica- a lo largo de las actividades planteadas.

PALABRAS CLAVES: modelos, modelización, ciencia escolar, contaminación atmosférica.

\section{ABSTRACT:}

The present investigation proposal takes in the perspective focused on the construction of models and modelling, in which teaching and learning are considered as a dynamic construction between the teacher and the students. The main purpose is to observe the evolution of the models constructed by the students through a sequence of teaching and learning in an eighth grade group of students from a school Tercer Milenio in Caldas, Antioquia. A qualitative methodology was used, specifically the instrumental study case. Student models related to scientific knowledge were built by the same students which ponder about the ecological balance - as in atmospheric pollution - along the proposed activities.

\footnotetext{
${ }^{1}$ Licenciado en Ciencias Naturales y Educación Ambiental, Universidad de Antioquia. Medellín Colombia. Correo electrónico: andres.gonzalez1@udea.edu.co

${ }^{2}$ Doctora en Didáctica de las Ciencias. Profesora, Universidad de Antioquia. Medellín - Colombia Correo electrónico: fanny.angulo@udea.edu.co
} 
Bio - grafía. Escritos sobre la Biología y su Enseñanza. ISSN 2027-1034

Edición Extraordinaria. p.p. 789 - 798

Memorias del IX Encuentro Nacional de Experiencias en Enseñanza de la Biología y la

Educación Ambiental. IV Congreso Nacional de Investigación en Enseñanza de la Biología.

KEY WORDS: models, modelling, students' model, school science, atmospheric pollution.

\section{INTRODUCCIÓN:}

En un mundo contemporáneo de retos y desafíos la educación ha pasado por múltiples transformaciones, pese a esto no maneja el mismo ritmo de la sociedad para asumirlos de la manera más pertinente, en donde la educación en ciencias toma un papel fundamental en el devenir de los problemas a afrontar. En concordancia con Izquierdo, Sanmartí \& Espinet "las ciencias constituyen una manera de pensar y de actuar con el objetivo de interpretar diferentes fenómenos e intervenir en ellos mediante un conjunto de conocimientos teóricos y prácticos estructurados" (1999, pág. 46), sin embargo la gran dificultad es que la enseñanza de las ciencias se ha caracterizado - incluso aún en la actualidad- por tener una forma dogmática y sin un criterio alguno lo cual ha sido una imagen transmitida tanto por la escuela como por los medios de comunicación en donde la ciencia es algo difícil de alcanzar.

Son varios los autores (véase por ejemplo; Izquierdo y Adúriz-Bravo (2003), Pujol (2003), García (2005), Chamizo (2009; 2010) y Gutiérrez (2001-2014)) se han preocupado por dejar de lado la manera tradicional y dogmática de la enseñanza de la ciencias basada solo en conceptos, en donde existe una relación vertical entre el docente y el estudiante y de igual manera con el conocimiento. Por tal motivo es imprescindible repensar este tipo de situaciones buscando una participación activa por parte del estudiante no solo en su aprendizaje sino también en la construcción del mismo. De acuerdo a esto es importante añadir que el docente no solo debe tener un profundo conocimiento disciplinar sino también ser crítico de lo que va a enseñar, debido a que en repetidas ocasiones tratan de impartir programas de ciencia sobrecargados y se presenta una indisposición general por no tener el tiempo necesario para explicarlo.

De acuerdo con Justi (2006) estamos educando estudiantes que van a vivir en un mundo sobre el cual sabemos bien poco, pero que, sin embargo, se va a caracterizar por rápidos cambios y probablemente será mucho más complejo e incierto del que experimentamos hoy. Por tal motivo las ciencias deben formar parte del currículo porque los ciudadanos del siglo XXI deberán analizar situaciones y tomar decisiones sobre asuntos que tienen que ver con conocimientos científicos o bien con habilidades técnicas.

Desde la perspectiva anterior y en concordancia con Justi:

[...] es incoherente pensar que la enseñanza de las ciencias se limite a la transmisión de una serie de conocimientos desvinculados y muchas veces obsoletos, y que el papel del alumno sea solamente acumular tales conocimientos. Esta perspectiva implica promover un modelo de enseñanza que ayude a las alumnas y alumnos a desarrollar una comprensión más coherente, flexible, sistemática y principalmente crítica. (2006, pág. 174). 


\title{
Bio - grafía. Escritos sobre la Biología y su Enseñanza. ISSN 2027-1034
}

\section{Edición Extraordinaria. p.p. 789 - 798}

\author{
Memorias del IX Encuentro Nacional de Experiencias en Enseñanza de la Biología y la \\ Educación Ambiental. IV Congreso Nacional de Investigación en Enseñanza de la \\ Biología.
}

Teniendo en cuenta estas consideraciones, los modelos y la modelización incluyen una forma de buscar una construcción que permita una verdadera relación entre la enseñanza, el aprendizaje y la evaluación, así como de centrar la atención durante su proceso, siendo facilitadora de la participación de los estudiantes llevando a una compresión de los modelos fundamentales de la ciencia así mismo a entender la naturaleza del conocimiento disciplinar.

Por tanto es importante resaltar los aportes de Gutiérrez $(2001,2014)$ quien brinda una definición coherente y clara sobre el concepto de modelo la cual se puede entender como "una representación de un sistema real o conjeturado, consistente en un conjunto de objetos con sus propiedades más sobresalientes enlistadas y un conjunto de reglas que declaran el comportamiento de dichos objetos" (2014, pág. 51). Esta autora también integra los componentes que hacen parte de un modelo, como lo son los ontológicos (ON) que constituyen un sistema de creencias del sujeto, el componente epistemológico (EP), que brinda criterios de verdad para validar las explicaciones anteriores, y psicológico (PSI) que mueve a las personas a modificar sus puntos de vista en tanto se replantea la causalidad. De allí surge la nominación ONEPSI.

\section{METODOLOGÍA:}

La metodología elegida para la investigación fue de corte cualitativo con un enfoque interpretativo, se eligió el estudio de caso, específicamente el estudio de caso instrumental debido a su interés se centra en investigar una situación paradójica, una necesidad de comprensión general mediante el estudio de un caso particular (Stake, 2005). La información registrada fue categorizada en consonancia con Gutiérrez (2001) y su propuesta del modelo ONEPSI; en donde se tomó como referencia el modelo estudiantil inicial de los estudiantes y el modelo curricular.

La secuencia de enseñanza y aprendizaje se diseñó teniendo en cuenta los aportes de Jorba \& Sanmarti (1996) y Pujol (2003) quienes mencionan que el modelo didáctico influye en la manera de enseñar un contenido con cierto grado de organización y planificación de las actividades mediante un diseño que fortalezca el aprendizaje, de acuerdo a esto también se tuvo presente el contexto del municipio ${ }^{3}$, en la cual se planteó una actividad para explorar los modelos estudiantiles iniciales, a partir de esta se obtuvo el modelo estudiantil inicial, posteriormente se revisaron los documentos de la institución y los lineamientos curriculares de ciencias naturales y educación ambiental para construir el modelo curricular.

\footnotetext{
${ }^{3}$ Caldas es un municipio que se encuentra hacia el sur del valle del Aburra, presenta una zona geográfica cercada por múltiples montañas formando una especie de barrera hacia los demás municipios aledaños por lo que los vientos y las lluvias se condensan constantemente en el municipio donde se manifiestan los mayores índices de contaminación en los últimos años.
} 
Bio - grafía. Escritos sobre la Biología y su Enseñanza. ISSN 2027-1034

Edición Extraordinaria. p.p. 789 - 798

Memorias del IX Encuentro Nacional de Experiencias en Enseñanza de la Biología y la

Educación Ambiental. IV Congreso Nacional de Investigación en Enseñanza de la Biología.

La secuencia contenía seis actividades en las que se incluyeron construcción de mapas mentales y conceptuales, exposiciones, video foro, elaboración de historietas sobre el problema planteado, entre otras, que tenía como hipótesis según la cual, las actividades promovían la modelización y los resultados permitían analizar los cambios en los modelos de los estudiantes.

\section{RESULTADOS Y DISCUSIÓN:}

A partir de las actividades realizadas de la secuencia de enseñanza y aprendizaje se registró la información para su posterior análisis. Para esto se utilizó como modelo el ONEPSI en el cual se registraron sus constituyentes ontológicos, epistemológicos y psicológicos. En un primer momento se realizó el MEi -Modelo estudiantil inicial, partiendo de los modelos explicitados por los estudiantes mediante la actividad de exploración, también se tuvo presente el MC - Modelo curricular, al que se pretende llevar a los estudiantes al final de la secuencia de enseñanza. Para cada una de las etapas se realizó una tabla donde se registraron sus respectivos constituyentes, se tomaron las tendencias de respuesta dadas por los estudiantes, de esta manera poder analizar así los cambios en los modelos de los estudiantes a lo largo de la implementación de la secuencia.

Durante la primera actividad (ver Tabla 1) de la secuencia de enseñanza y aprendizaje, se puede evidenciar que la mayoría de las respuestas se agrupan y tienden hacia los constituyentes ontológicos y epistemológicos, algunos pocos se ubican en los psicológicos. Con respecto a los enunciados se resalta una construcción de argumentos muy básicos $\mathrm{Si}$ bien los estudiantes relacionan algunas causas, aún siguen siendo explicaciones inmediatas donde no se explican procesos propios de las ciencias ni tampoco la incorporación de conceptos.

Durante el desarrollo de las demás actividades y para el caso de la fase de introducción de nuevos conocimientos (ver Tabla 2) es posible afirmar que fue significativa para los estudiantes, debido a que subyace un acercamiento de los estudiantes a los conceptos del modelo curricular, además de propiciar un espacio que les permitió comprender diversos aspectos de la problemática tratada y conocer así los puntos de vista y cambios en los modelos construidos a partir de sus propias construcciones y autorregulación.

En relación con la apropiación de conceptos y de conocimiento de las ciencias naturales y desde un punto de vista disciplinar, es importante prestar atención a las relaciones y sustentos utilizados de los estudiantes, por ejemplo en la elaboración de la historieta conceptos como Aire se vuelven a presentar pero en este caso sus propiedades con capa gaseosa que envuelve al planeta Tierra., y una explicación de causalidad que es: el aire se contamina por la emisión de humo, emisión de aerosoles, el aire se contamina por el dióxido de azufre, monóxido de carbono y dióxido de carbono. 
Bio - grafía. Escritos sobre la Biología y su Enseñanza. ISSN 2027-1034

Edición Extraordinaria. p.p. 789 - 798

Memorias del IX Encuentro Nacional de Experiencias en Enseñanza de la Biología y la

Educación Ambiental. IV Congreso Nacional de Investigación en Enseñanza de la Biología.

A continuación se presentan algunos de los fragmentos de los modelos construidos por los estudiantes sintetizados en tablas ${ }^{4}$

\section{TABLA 1.MODELO ESTUDIANTIL INICIAL}

\section{ACTIVIDAD DE EXPLORACIÓN}

Durante esta actividad los estudiantes respondieron y elaboraron el modelo inicial a partir de un cuestionario y una noticia sobre la problemática de la contaminación atmosférica, a partir de las cuales se construyó el modelo estudiantil inicial.

\begin{tabular}{|c|c|c|c|c|c|}
\hline \multicolumn{2}{|c|}{ Constituyentes Ontológicos } & \multicolumn{2}{|c|}{ Constituyentes Epistemológicos } & \multirow{2}{*}{\multicolumn{2}{|c|}{$\begin{array}{c}\text { Constituyentes Psicológicos } \\
\text { (Causalidad) }\end{array}$}} \\
\hline \multicolumn{4}{|c|}{ Enunciados Legales } & & \\
\hline Entidades & Propiedades & Relaciones & \multicolumn{3}{|c|}{ Inferencias } \\
\hline & & Describir & Predecir & Explicar & Inferencia \\
\hline \multicolumn{6}{|c|}{ Dibujo: Procesos de contaminación (en la noticia) } \\
\hline Agua & $\begin{array}{l}\text { El agua se contamina } \\
\text { con basuras. }\end{array}$ & & & & \\
\hline Arboles & $\begin{array}{ll}\text { Los árboles } & \text { se } \\
\text { destruyen por la } \\
\text { contaminación }\end{array}$ & $\begin{array}{l}\text { Hay un maltrato por } \\
\text { las zonas con } \\
\text { árboles. }\end{array}$ & & & $\begin{array}{l}\text { La } \\
\text { contaminación } \\
\text { es causa de la } \\
\text { destrucción } \\
\text { de le los } \\
\text { árboles. }\end{array}$ \\
\hline Personas & $\begin{array}{l}\text { Las personas mueren } \\
\text { por la contaminación. }\end{array}$ & & & $\begin{array}{l}\text { Las personas usan } \\
\text { tapabocas por la }\end{array}$ & \\
\hline
\end{tabular}

\footnotetext{
${ }^{4}$ Se presentan solo la actividad de exploración y de introducción de nuevos de conocimientos para mostrar la herramienta ONEPSI. Las demás tablas se encuentran en la investigación principal que tiene el mismo título de este artículo.
} 
Bio - grafía. Escritos sobre la Biología y su Enseñanza. ISSN 2027-1034

Edición Extraordinaria. p.p. $789-798$

Memorias del IX Encuentro Nacional de Experiencias en Enseñanza de la Biología y la

Educación Ambiental. IV Congreso Nacional de Investigación en Enseñanza de la Biología.

\begin{tabular}{|c|c|c|c|}
\hline & & & contaminación. \\
\hline Aire & El aire se contamina. & $\begin{array}{l}\text { El aire se vuelve } \\
\text { más oscuro. }\end{array}$ & $\begin{array}{l}\text { Por la } \\
\text { contaminación del } \\
\text { aire se pueden } \\
\text { ocasionar muertes } \\
\text { de seres vivos. }\end{array}$ \\
\hline Fábricas & $\begin{array}{l}\text { Las fábricas } \\
\text { contaminan el aire. }\end{array}$ & $\begin{array}{lr}\text { Las } & \text { fábricas } \\
\text { contaminan el aire, } \\
\text { las fuentes de agua, } \\
\text { destruyen los } \\
\text { árboles. }\end{array}$ & \\
\hline
\end{tabular}


Bio - grafía. Escritos sobre la Biología y su Enseñanza. ISSN 2027-1034

Edición Extraordinaria. p.p. 789 - 798

Memorias del IX Encuentro Nacional de Experiencias en Enseñanza de la Biología y la

Educación Ambiental. IV Congreso Nacional de Investigación en Enseñanza de la Biología.

\begin{tabular}{|c|c|c|c|c|c|}
\hline \multicolumn{6}{|c|}{$\begin{array}{l}\text { TABLA2.ACTIVID AD DE INTRODUCCION DE NUEVOS CONOCIMIENTOS (ELABORACION HISTORIETA), } \\
\text { En esta actividad los estudiantes realizaron una historieta que relacionara los temas vistos durante las explicaciones } \\
\text { en clase y dar una posible solución al problema de la contaminación atmosférica. }\end{array}$} \\
\hline Constituye & tes Ontológicos & \multicolumn{2}{|c|}{ Constituyentes Epistemológicos } & \multicolumn{2}{|c|}{ Constituyentes P sicológicos } \\
\hline \multicolumn{4}{|c|}{ Enun ciados Legales } & \multicolumn{2}{|c|}{ (Causalidad) } \\
\hline \multirow[t]{2}{*}{ Entidades } & Propiedades & Relaciones & \multicolumn{3}{|c|}{ Inferencias } \\
\hline & & Describir & Predecir & Explicar & Inferencia \\
\hline \multicolumn{6}{|c|}{ ELABORACION HISTORIETA } \\
\hline Aire & $\begin{array}{l}\text { Capa gaseosa } \\
\text { que envuelve al } \\
\text { planeta tierra. }\end{array}$ & $\begin{array}{l}\text { El aire se contamina por } \\
\text { la emisión de humo, } \\
\text { emisión de aerosoles, } \\
\text { malos olores, radiación } \\
\text { atómica. } \\
\text { El aire se contamina por } \\
\text { el dióxido de azufre, } \\
\text { monóxido de carbono y } \\
\text { dióxido de carbono. } \\
\text { Fumar afecta la calidad } \\
\text { del aire. }\end{array}$ & $\begin{array}{l}\text { La deforestación, la } \\
\text { quema de } \\
\text { combustibles } \\
\text { fósiles, la quema } \\
\text { de madera y el } \\
\text { humo de las } \\
\text { fábricas. }\end{array}$ & $\begin{array}{l}\text { Cuidar el aire es } \\
\text { responsabilidad de } \\
\text { todos y velar por el } \\
\text { cuidado de este porque } \\
\text { el planeta es de todos. } \\
\text { Los desechos de las } \\
\text { fábricas son un gran } \\
\text { contaminante si no son } \\
\text { tratados } \\
\text { adecuadamente. }\end{array}$ & $\begin{array}{l}\text { La contaminación } \\
\text { de aire puede } \\
\text { provocar } \\
\text { enfermedades } \\
\text { respiratorias como } \\
\text { el asma, la } \\
\text { bronquitis } \\
\text { enfermedades de la } \\
\text { piel, además afecta } \\
\text { el crecimiento de } \\
\text { las plantas. }\end{array}$ \\
\hline Ambiente & & $\begin{array}{l}\text { El consumo desmedido } \\
\text { e innecesario afecta el } \\
\text { equilibrio del ambiente. } \\
\text { Los mares y ríos se ven } \\
\text { afectados por la } \\
\text { contaminación. }\end{array}$ & $\begin{array}{l}\text { La desaparición de } \\
\text { bosques es causa } \\
\text { de la } \\
\text { contaminación } \\
\text { El ambiente sufre } \\
\text { contaminación } \\
\text { constante por las } \\
\text { operaciones } \\
\text { industriales que se }\end{array}$ & $\begin{array}{l}\text { Ahorrar agua, reutilizar. } \\
\text { Sembrar árboles, utilizar } \\
\text { materiales } \\
\text { biodegradables. Por } \\
\text { culpa de la } \\
\text { contaminación se ha } \\
\text { perdido de una fuente } \\
\text { natural y necesaria para } \\
\text { la vida humana, lo que }\end{array}$ & $\begin{array}{l}\text { Es necesario } \\
\text { conservar el suelo y } \\
\text { el agua para } \\
\text { asegurar un futuro. }\end{array}$ \\
\hline
\end{tabular}


Bio - grafía. Escritos sobre la Biología y su Enseñanza. ISSN 2027-1034

Edición Extraordinaria. p.p. 789 - 798

Memorias del IX Encuentro Nacional de Experiencias en Enseñanza de la Biología y la Educación Ambiental. IV Congreso Nacional de Investigación en Enseñanza de la Biología.

\begin{tabular}{|c|c|c|c|c|}
\hline & & $\begin{array}{lr}\text { realizan } & \text { con } \\
\text { productos } & \text { tóxicos } \\
\text { como la minería, } \\
\text { metales pesados, } \\
\text { fabricación } \quad \text { de } \\
\text { plaguicidas } \\
\text { pesticidas. }\end{array}$ & $\begin{array}{l}\text { puede } \\
\text { enfermedades como la } \\
\text { cólera, la hepatitis, el } \\
\text { tifus. }\end{array}$ & \\
\hline Atmósfera & 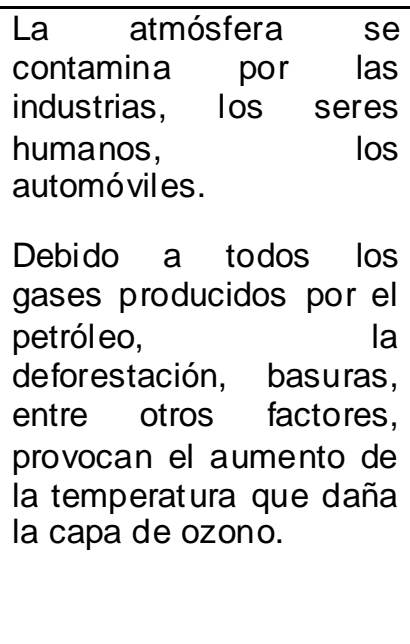 & $\begin{array}{l}\text { La contaminación } \\
\text { atmosférica se por } \\
\text { la mezcla de gases } \\
\text { tóxicos para los } \\
\text { seres vivos. }\end{array}$ & $\begin{array}{l}\text { Generalmente los gases } \\
\text { son liberados por los } \\
\text { tubos de eliminación a } \\
\text { la atmósfera que son el } \\
\text { dióxido de carbono, } \\
\text { metano, y óxidos. Los } \\
\text { gases que los carros } \\
\text { liberan en su proceso } \\
\text { de combustión son tan } \\
\text { fuertes que últimamente } \\
\text { se están generando } \\
\text { grandes cantidades de } \\
\text { Co2 Cada vez que } \\
\text { contamínanos } \\
\text { destruye la capa de } \\
\text { ozono. }\end{array}$ & $\begin{array}{l}\text { No habría tantas } \\
\text { enfermedades sin } \\
\text { tanta } \\
\text { contaminación. }\end{array}$ \\
\hline
\end{tabular}


Bio - grafía. Escritos sobre la Biología y su Enseñanza. ISSN 2027-1034

Edición Extraordinaria. p.p. 789 - 798

Memorias del IX Encuentro Nacional de Experiencias en Enseñanza de la Biología y la

Educación Ambiental. IV Congreso Nacional de Investigación en Enseñanza de la Biología.

\section{CONCLUSIONES:}

- Respecto a los modelos construidos por los estudiantes en el contexto del fenómeno de valor educativo de la contaminación atmosférica, se encontraron varios cambios que se acercaban al modelo curricular en concordancia con datos y conocimientos de la biología y química, sin embargo los cambios en los modelos no se presentaron en todos los estudiantes debido a que variaba en el uso de los argumentos y las fuentes de información durante las actividades propuestas.

- La secuencia de enseñanza y aprendizaje contribuyó con las posibilidades que tienen los estudiantes de manifestar posturas sobre una problemática ambiental que se encuentra constantemente en la vida cotidiana y que es de carácter social y de interés en el contexto colombiano. En algunas de las intervenciones de los estudiantes, es posible inferir, que más allá del uso de entidades, se manifiestan también argumentos propositivos que aluden a posibles soluciones al problema de la contaminación atmosférica, aportando así al aprendizaje de entidades desde una perspectiva diferente a la enseñanza tradicional.

\section{BIBLIOGRAFÍA:}

Chamizo, J.A. (2009). Modelos y modelaje. Una primera descripción para su uso en las aulas. Enseñanza de las Ciencias, Número Extra VIII Congreso Internacional sobre Investigación en Didáctica de las Ciencias, Barcelona, 2057-2061 http://ensciencias.uab.es/congreso09/numeroextra/art-2057-2061.pdf

Chamizo, J.A. (2010). Una tipología de los modelos para la enseñanza de las ciencias. Revista Eureka sobre Enseñanza y Divulgación de las Ciencias, 7, 26-41. Recuperado de http://www.redalyc.org/articulo.oa?id=92013011003

Gutiérrez, R. (2001). Mental Models and the fine structure of Conceptual Change. En: Pinto, R. \& Surinach, S. (eds). Physics Teacher Education Beyond 2000. Elsevier Editions. París, 35-44.

Gutiérrez, R. (2014). Lo que los profesores de ciencia conocen y necesitan conocer acerca de los modelos: aproximaciones y alternativas. Biografía. Escritos sobre la biología y su enseñanza. 7(13), $37-66$.

Izquierdo, M., \& Adúriz-Bravo, A. (2003). Epistemological Foundations of School Science. Science \& Education(12), 27-43.

Izquierdo, M.; Sanmartí, N.; Espinet, M.; García, M.P. \& Pujol, R.M. (1999). Caracterización y fundamentación de la ciencia escolar: Enseñanza de las Ciencias. Núm. Extra, 79-92.

Jorba, J. \& Sanmartí, N. (1996).Enseñar, aprender y evaluar: un proceso de regulación continua: propuestas didácticas para las áreas de ciencias de la naturaleza y matemáticas. Ministerio de educación y cultura. Barcelona: España. 
Bio - grafía. Escritos sobre la Biología y su Enseñanza. ISSN 2027-1034

Edición Extraordinaria. p.p. 789 - 798

Memorias del IX Encuentro Nacional de Experiencias en Enseñanza de la Biología y la Educación Ambiental. IV Congreso Nacional de Investigación en Enseñanza de la Biología.

Justi, R. (2006). La enseñanza de la ciencia basada en la elaboración de modelos, Enseñanza de las ciencias: Revista de investigación y experiencias didácticas, 24, 173184.

Pujol, R.M. (2003).Didáctica de las Ciencias en la Educación Primaria. Síntesis: Madrid. Stake, R. (2005). Investigación con estudio de casos. Madrid: Morata. 